\title{
Remissão de hipertensão arterial sistêmica, Diabetes mellitus tipo II e dislipidemia após cirurgia bariátrica: um estudo longitudinal
}

\author{
Remission of systemic arterial hypertension, type II Diabetes mellitus and dyslipidemia after \\ bariatric surgery: a longitudinal study \\ Remisión de la hipertensión arterial sistémica, Diabetes mellitus tipo II y dislipidemia tras la cirugía \\ bariátrica: un estudio longitudinal
}

Recebido: 08/09/2021 | Revisado: 16/09/2021 | Aceito: 21/09/2021 | Publicado: 23/09/2021

\author{
Alex Cristiano Rech \\ ORCID: https://orcid org/0000-0001-5882-7180 \\ Centro Universitário da Fundação Assis Gurgacz, Brasil \\ E-mail: alexcristianorech@hotmail.com \\ Helin Minoru Matsumoto \\ ORCID: https://orcid.org/0000-0001-6184-8775 \\ Centro Universitário da Fundação Assis Gurgacz, Brasil \\ E-mail: minoru@gastro.com.br
}

\begin{abstract}
Resumo
A gastroplastia redutora, também conhecida como cirurgia bariátrica, é uma operação recomendada para pacientes com índice de massa corporal (IMC) $>40 \mathrm{~kg} / \mathrm{m}^{2}$, independentemente da presença de comorbidades e IMC entre 35 e $40 \mathrm{~kg} / \mathrm{m}^{2}$ na presença de doenças associadas. Cirurgias bariátricas podem ser divididas em restritivas ou mistas. As restritivas ocorrem quando apenas o estômago é modificado. Nas cirurgias mistas, além do estômago, há alteração no intestino do paciente. Em ambos os casos ocorre expressiva redução no peso após operação. Este estudo buscou avaliar a remissão de doenças como hipertensão arterial sistêmica (HAS), diabetes mellitus tipo 2 (DM II) e dislipidemia em até cinco anos após operação e após este período em 53 pacientes acompanhados ambulatorialmente em uma clínica de gastroenterologia no oeste do Paraná. Houve redução na prevalência das três patologias estudadas no período até cinco anos após a operação $(\mathrm{p}<0,001)$ e após cinco anos $(\mathrm{p}<0,001)$. Portanto, evidencia-se a efetividade da cirurgia bariátrica na redução da prevalência de doenças decorrentes da obesidade.
\end{abstract}

Palavras-chave: Gastroplastia; Cirurgia bariátrica; Diabetes Mellitus; Hipertensão.

\begin{abstract}
Reductive gastroplasty, also known as bariatric surgery, is recommended for patients with body mass index (BMI) $>40$ $\mathrm{kg} / \mathrm{m}^{2}$, regardless of the presence of comorbidities and BMI between 35 and $40 \mathrm{~kg} / \mathrm{m}^{2}$ if associated diseases presented. Bariatric surgeries can be divided into restrictive or mixed. The restrictive ones occur when only the stomach is modified. In mixed surgeries, there is a change in patient's intestine and stomach. Both operations reduce the patient's weight. This study sought to assess the remission of diseases such as systemic arterial hypertension, type two diabetes mellitus and dyslipidemia within five years after the operation and after this period in 53 patients of a gastroenterology clinic in west Parana. A reduction in the prevalence of all three studied pathologies was observed in the period up to five years after the operation $(p<0,001)$ and after five years $(p<0,001)$. Therefore, the effectiveness of bariatric surgery in reduction of the prevalence of diseases resulting from obesity is evident.
\end{abstract}

Keywords: Gastroplasty; Bariatric surgery; Diabetes Mellitus; Hypertension.

\section{Resumen}

La gastroplastia de reducción, también conocida como cirugía bariátrica, es una operación recomendada para pacientes con un índice de masa corporal (IMC) $>40 \mathrm{~kg} / \mathrm{m} 2$, independientemente de la presencia de comorbilidades, y IMC entre 35 y $40 \mathrm{~kg} / \mathrm{m} 2$ en presencia de enfermedades asociadas. Las cirugías bariátricas pueden dividirse en restrictivas o mixtas. Las restrictivas se producen cuando sólo se modifica el estómago. En las cirugías mixtas, además del estómago, hay una alteración en el intestino del paciente. En ambos casos se produce una importante reducción de peso tras la cirugía. Este estudio buscó evaluar la remisión de enfermedades como la hipertensión arterial sistémica (HAS), la diabetes mellitus tipo 2 (DM II) y la dislipidemia en los cinco años posteriores a la cirugía y después de este período en 53 pacientes controlados en una clínica ambulatoria del oeste de Paraná. Hubo una reducción en la prevalencia de las tres patologías estudiadas en el período hasta cinco años después de la cirugía $(\mathrm{p}<0,001)$ y después de cinco años $(\mathrm{p}<0,001)$. Por lo tanto, es evidente la eficacia de la cirugía bariátrica para reducir la prevalencia de las enfermedades derivadas de la obesidad.

Palabras clave: Gastroplastia; Cirugía bariátrica; Diabetes Mellitus; Hipertensión. 


\section{Introdução}

A gastroplastia, também conhecida como cirurgia bariátrica, é uma operação recomendada para pacientes com índice de massa corporal (IMC) $>40 \mathrm{~kg} / \mathrm{m}^{2}$, independentemente da presença de comorbidades (hipertensão arterial, dislipidemia, diabetes tipo 2, apneia do sono, artropatia, entre outras) e IMC entre 35 e 40kg/m² na presença de doenças associadas (Marcelino e Patrício, 2011). A redução no peso após cirurgia bariátrica é expressiva em diversos estudos (O` Brien et al, 2018; Wolfe et al, 2016). Como a obesidade apresenta prevalência crescente no país, a incidência de cirurgias bariátricas cresce anualmente no Brasil. Foram realizadas cerca de 100.000 operações em 2016, com aumento estimado de $300 \%$ na comparação com 2006. (Carvalho e Rosa, 2019).

Cirurgias bariátricas podem ser dividas em restritivas ou mistas. As restritivas ocorrem quando apenas o estômago é modificado, reduzindo espaço para o alimento, provocando sensação de saciedade com menor quantidade de alimento. Um exemplo é a gastrectomia vertical ou sleeve, que atualmente é a técnica mais realizada no mundo. A cirurgia consiste inicialmente em mobilizar completamente a grande curvatura do estômago até proximalmente ao ângulo de Hiss. Após a exposição da crura diafragmática esquerda e visualização do estômago posterior, a gastrectomia vertical começa distante 4-6 cm do piloro com a criação de um estômago tubularizado medindo entre 34-40 Fr de diâmetro cuidando para não estreitar a incisura, espiralizar o neoestômago ou deixar muito fundo gástrico (Zeve et al, 2012; Chung et al, 2018).

Os benefícios associados a cirurgia bariátrica descritos na literatura médica são remissão do diabetes mellitus tipo 2 (DM II) e hipertensão arterial sistêmica (HAS), melhor controle glicêmico, perda de peso (que reduz quadros de apneia-hipopneia em quadros de apneia obstrutiva do sono), redução de dores causadas por osteoartrite, incontinência urinária e de diversos cânceres associados com a obesidade (Arterburn et al, 2020).

Em metanálise realizada por Cardoso et al (2017), pacientes submetidos a gastroplastia apresentam significante redução de $41 \%$ no risco relativo de óbito em longo prazo por todas as causas (IC 95\% 0,52-0,67; p<0,001). Além disso, foi demonstrado que esta operação também reduz mortalidade por câncer $(\mathrm{p}<0,001)$ e por doenças cardiovasculares $(\mathrm{p}<0,001)$.

Dessa forma, este estudo tem o objetivo de descrever os benefícios da cirurgia bariátrica na forma de remissão da HAS, DM II e Dislipidemia durante os cinco primeiros anos após gastroplastia e após este período, na comparação com prevalência destas doenças no pré-operatório. Visando avaliar a evolução destas doenças, foram estudados prontuários de pacientes em acompanhamento ambulatorial em uma clínica privada de gastroenterologia sediada no oeste do Paraná.

\section{Metodologia}

Trata-se de um estudo quantitativo observacional com abordagem longitudinal, realizada através da análise de prontuários médicos obtidos de uma clínica privada de gastroenterologia sediada no oeste do Paraná. Foram estudados cinquenta e três pacientes com idade superior a 18 anos, que realizaram cirurgia bariátrica entre os anos de 2014 e 2015 . A pesquisa foi aprovada pelo Comitê de Ética em Pesquisas Com Seres Humanos do Centro Universitário da Fundação Assis Gurgacz com número CAAE 3867620.0.0000.5219. A base metodológica utilizada no estudo foi estruturada pelo trabalho de Pereira et al (2018).

Foram buscados nos prontuários a idade do paciente no momento da cirurgia, sexo, tipo de técnica e referências ao diagnóstico de Hipertensão Arterial Sistêmica (HAS), Diabetes Mellitus Tipo II (DM II) e Dislipidemia no período préoperatório, durante os primeiros cinco anos pós-operatórios e após cinco anos da cirurgia. Foi avaliado, por meio de testes estatísticos, se houve redução na prevalência de HAS, DM II e dislipidemia nos primeiros cinco anos ou após este período na comparação com pré-operatório. 
Os critérios de inclusão foram idade $\geq 18$ anos, realização da cirurgia entre 2014-2015 e acompanhamento por no mínimo cinco anos (60 meses) após o procedimento. Desta forma, foram excluídos do estudo os pacientes com idade inferior a 18 anos, que foram operados antes de 2014 e após 2015 além daqueles que houve perda do seguimento nos 60 meses subsequentes a cirurgia.

As análises estatísticas foram realizadas no software RStudio na versão 1.4.1103 utilizando teste Qui-Quadrado e Fisher. Para a associação estatística ser considerada válida foi utilizado nível de significância $(\alpha)$ de 0,05 . Portanto, foram consideradas estatisticamente relevantes associações cujo p-valor foi inferior a 0,05 .

\section{Resultados e Discussão}

A maioria dos participantes desta pesquisa são mulheres (83\%) e apresentam idade entre 21 até 50 anos $(56,6 \%)$. A maioria dos pacientes foi operado utilizando a técnica de bypass gástrico (82,4\%). A Tabela 1 resume as frequências absoluta e relativa das principais características dos participantes da pesquisa.

Tabela 1: Descrição do sexo, idade e técnica cirúrgica utilizada na gastroplastia de pacientes obesos atendidos em uma clínica privada no oeste do Paraná

\begin{tabular}{lcc}
\hline Característica & $\begin{array}{c}\text { Frequência } \\
\text { Absoluta }\end{array}$ & $\begin{array}{c}\text { Frequência } \\
\text { Relativa }\end{array}$ \\
\hline Sexo & 44 & $83 \%$ \\
Feminino & 9 & $17 \%$ \\
Masculino & & \\
Idade & 3 & $5,7 \%$ \\
$21-30$ anos & 14 & $26,4 \%$ \\
$31-40$ anos & 13 & $24,5 \%$ \\
$41-50$ anos & 18 & $34 \%$ \\
$51-60$ anos & 5 & $9,4 \%$ \\
$61-70$ anos & & \\
Técnica utilizada & 42 & $82,4 \%$ \\
Bypass gástrico & 9 & $17,6 \%$ \\
Sleeve (gastrectomia vertical) & 9 \\
\hline
\end{tabular}

Fonte: Autores (2021).

$\mathrm{Na}$ Tabela 2 verifica-se que a cirurgia bariátrica foi efetiva em reduzir significativamente a prevalência de doenças como Hipertensão Arterial Sistêmica (HAS), Diabetes Mellitus Tipo II (DM II) e quadros de dislipidemia. Com relação a HAS, 64,2\% pacientes estavam diagnosticados com esta patologia, havendo redução para 1,9\% dentro de cinco após a cirurgia $(\mathrm{p}<0,001)$ e $3,8 \%$ após cinco anos da operação ( $\mathrm{p}<0,001)$. Esses resultados são compatíveis com outros estudos que também demonstraram remissão da hipertensão arterial em pacientes que foram submetidos a gastroplastia (Schiavon et al, 2017).

Como cirurgia bariátrica é recomendada principalmente para pacientes obesos, elevada prevalência de DM II é esperada nestes pacientes devido ao mecanismo de resistência insulínica associado a síndrome metabólica (Cordero e Oben, 2017; Jung e Choi, 2014). Foi demonstrado que o controle glicêmico propiciado pela gastroplastia foi superior ao obtido em métodos não invasivos (Maggard-Gibbons et al, 2013). Neste trabalho, a prevalência de DM II foi reduzida de 41,5\% no pré-operatório para 
$1,9 \%$ durante cinco anos após cirurgia ( $<<0,001)$ e 3,8\% para cinco anos após ( $\mathrm{p}<0,001)$ (Tabela 2). Em artigo escrito por Affinati et al (2019) foi descrito remissão expressiva de DM II, de 33\% até 90\% após realização de gastroplastia.

Além da redução da prevalência de HAS e DM II a cirurgia bariátrica foi efetiva em reduzir a prevalência de dislipidemia, que inclui grande diversidade de anormalidade nos lipídios que pode ser representada por combinação de aumento no colesterol total ( $\geq 24 \mathrm{mg} / \mathrm{dL}$ ), LDL-C (>160 mg/dL) e triglicerídeos (>200 mg/dL) ou redução no HDL-C (<40mg/dL) (Kopin e Lowenstein, 2017). Como a dislipidemia é fator de risco para doenças como doença arterial coronariana (Kalita et al, 2018) a redução da prevalência de dislipidemia, associado com menor incidência de hipertensão e diabetes mellitus tipo 2, propiciado pela gastroplastia redutora pode estar associada a redução da mortalidade (Wiggins et al, 2020).

Um dos fatores que podem explicar sucesso da cirurgia bariátrica no contexto de redução da HAS, DM II e Dislipidemia neste estudo é idade relativamente jovem dos pacientes (média de 46,49 anos na operação). Outros trabalhos mostraram maiores benefícios associados a gastroplastia em pacientes mais jovens (Cooiman et al, 2019; Chikungwo et al, 2010; Coleman et al, 2016).

Tabela 2: Prevalência de Hipertensão Arterial Sistêmica (HAS), Diabetes Mellitus Tipo II (DM II) e dislipidemia no pré-operatório, durante cinco após a cirurgia e após este período em pacientes obesos que realizaram gastroplastia redutora.

\begin{tabular}{lcc}
\hline Doença & Prevalência & Valor p \\
\hline Hipertensão Arterial Sistêmica (HAS) & & \\
$\quad$ Pré operatório & $64,2 \%$ & Referência \\
$\quad$ Durante cinco anos no pós-operatório & $1,9 \%$ & $<0,001$ \\
Após cinco anos do pós-operatório & $3,8 \%$ & $<0,001$ \\
Diabetes Mellitus Tipo II (DM II) & & \\
$\quad$ Pré operatório & $41,5 \%$ & Referência \\
$\quad$ Durante cinco anos no pós-operatório & $1,9 \%$ & $<0,001$ \\
Após cinco anos do pós-operatório & $3,8 \%$ & $<0,001$ \\
Dislipidemia & & \\
Pré operatório & $73,6 \%$ & Referência \\
Durante cinco anos no pós-operatório & $1,9 \%$ & $<0,001$ \\
Após cinco anos do pós-operatório & $1,9 \%$ & $<0,001$ \\
\hline
\end{tabular}

Fonte: Autores (2021).

\section{Conclusão}

Este estudo mostrou que a gastroplastia redutora apresenta grande efetividade na redução da prevalência de doenças decorrentes da obesidade, como Hipertensão Arterial Sistêmica (HAS), Diabetes Mellitus Tipo II (DM II) e dislipidemia (Tabela 2). Na comparação com outros trabalhos, a redução foi ainda mais expressiva, possivelmente decorrente da amostra deste estudo ser mais jovem.

Dessa forma, respeitando os critérios clínicos de recomendação, a cirurgia bariátrica é uma das principais intervenções que reduzem mortalidade a longo prazo em pacientes obesos (Sjostrom et al, 2007). Isso possivelmente está associado a expressiva redução na prevalência de diversas doenças associadas a mortalidade (Sheng et al, 2017) em pacientes obesos, conforme demonstrado na Tabela 2.

Futuros trabalhos podem avaliar se a eficácia da gastroplastia redutora na redução da prevalência de doenças decorrentes da obesidade ocorre também em estudos com maior rigor metodológico, como em pesquisas realizadas em mais de uma instituição (multicêntricas) e randomizadas. 


\section{Referências}

Affinati, A. H., Esfandiari, N. H., Oral, E. A., \& Kraftson, A. T. (2019). Bariatric surgery versus medications in the treatment of type 2 diabetes. Current Diabetes Reports, 19(156), 1-10.

Arterburn, D. E., Telem, D. A., Kushner, R. F., \& Courcoulas, A. P. (2020). Benefits and Risks of Bariatric Surgery in Adults: A Review. JAMA - Journal of the American Medical Association, 324(9), 879-887. https://doi.org/10.1001/jama.2020.12567

Balsiger, B. M., Kennedy, F. P., Abu-Lebdeh, H. S., Collazo-Clavell, M., Jensen, M. D., O’Brien, T., Hensrud, D. D., Dinneen, S. F., Thompson, G. B., Que, F. G., Williams, D. E., Clark, M. M., Grant, J. E., Frick, M. S., Mueller, R. A., Mai, J. L., \& Sarr, M. G. (2000). Prospective Evaluation of Roux-en-Y Gastric Bypass as Primary Operation for Medically Complicated Obesity. Mayo Clinic Proceedings, 75(7), 673-680. https://doi.org/10.4065/75.7.673

Cardoso, L., Rodrigues, D., Gomes, L., \& Carrilho, F. (2017). Short- and long-term mortality after bariatric surgery: A systematic review and meta-analysis Diabetes, Obesity and Metabolism, 19(9), 1223-1232. https://doi.org/10.1111/dom.12922

Carvalho, A. da S., \& Rosa, R. D. S. (2019). Cirurgias bariátricas realizadas pelo Sistema Único de Saúde no período 2010-2016: estudo descritivo das hospitalizações no Brasil. Epidemiologia e Servicos de Saude : Revista Do Sistema Unico de Saude Do Brasil, 28(1), e2018260. https://doi.org/10.5123/S167949742019000100023

Chikunguwo, S. M., Wolfe, L. G., Dodson, P., Meador, J. G., Baugh, N., Clore, J. N., Kellum, J. M., \& Maher, J. W. (2010). Analysis of factors associated with durable remission of diabetes after Roux-en-Y gastric bypass. Surgery for Obesity and Related Diseases, 6(3), 254-259. https://doi.org/10.1016/j.soard.2009.11.003

Chung, A. Y., Thompson, R., Overby, D. W., Duke, M. C., \& Farrell, T. M. (2018). Sleeve Gastrectomy: Surgical Tips. Journal of Laparoendoscopic and Advanced Surgical Techniques, 28(8), 930-937. https://doi.org/10.1089/lap.2018.0392

Coleman, K. J., Haneuse, S., Johnson, E., Bogart, A., Fisher, D., O’Connor, P. J., Sherwood, N. E., Sidney, S., Theis, M. K., Anau, J., Schroeder, E. B., O’Brien, R., \& Arterburn, D. (2016). Long-term microvascular disease outcomes in patients with type 2 diabetes after bariatric surgery: Evidence for the legacy effect of surgery. Diabetes Care, 39(8), 1400-1407. https://doi.org/10.2337/dc16-0194

Cooiman, M., Mol, B., Aarts, E., Janssen, I., Hazebroek, E., \& Berends, F. (2019). Weight loss, reduction of comorbidities and quality of life after bariatric surgery in the elderly. Obesity Surgery, 29(6), 1851-1857. https://doi.org/10.1007/s11695-019-03781-z.

Cordero, P., Li, J., \& Oben, J. A. (2017). Bariatric surgery as a treatment for metabolic syndrome. Journal of the Royal College of Physicians of Edinburgh, 47(4), 364-368. https://doi.org/10.4997/JRCPE.2017.414

Hydock, C. M. (2005). A brief overview of bariatric surgical procedures currently being used to treat the obese patient. Critical Care Nursing Quarterly, 28(3), 217-226. https://doi.org/10.1097/00002727-200507000-00002

Jung, U. J., \& Choi, M. S. (2014). Obesity and its metabolic complications: The role of adipokines and the relationship between obesity, inflammation, insulin resistance, dyslipidemia and nonalcoholic fatty liver disease. International Journal of Molecular Sciences, 15(4), 6184-6223. https://doi.org/10.3390/ijms15046184

Kalita, S., Khandelwal, S., Madan, J., Pandya, H., Sesikeran, B., \& Krishnaswamy, K. (2018). Almonds and cardiovascular health: A review. Nutrients, 10(4), 1-10. https://doi.org/10.3390/nu10040468

Kopin, L., \& Lowenstein, C. J. (2017). Dyslipidemia. Annals of Internal Medicine, 167(11), ITC81-ITC96. https://doi.org/10.7326/aitc201712050

Maggard, M. A., Shugarman, L. R., Suttorp, M., Maglione, M., Sugarman, H. J., Livingston, E. H., Nguyen, N. T., Li, Z., Mojica, W. A., Hilton, L., \& Rhodes, S. (2005). Clinical Guidelines Meta-Analysis: Surgical Treatment of Obesity. Annals of Internal Medicine.

Maggard-Gibbons, M. A., Maglione, M., Livhits, M., Ewing, B., Maher, A. R., Hu, J., Li, Z., \& Shekelle, P. G. (2013). Bariatric surgery for weight loss and glycemic control in nonmorbidly obese adults with diabetes a systematic review. JAMA - Journal of the American Medical Association, 309(21), 2250-2261. https://doi.org/10.1001/jama.2013.4851

Marcelino, L. F., \& Patrício, Z. M. (2011). The complexity of obesity and life after bariatric surgery: A public health issue. Ciencia e Saude Coletiva, 16(12), 4767-4776. https://doi.org/10.1590/s1413-81232011001300025

O’Brien, P. E., Hindle, A., Brennan, L., Skinner, S., Burton, P., Smith, A., Crosthwaite, G., \& Brown, W. (2018). Long-Term Outcomes After Bariatric Surgery: a Systematic Review and Meta-analysis of Weight Loss at 10 or More Years for All Bariatric Procedures and a Single-Centre Review of 20-Year Outcomes After Adjustable Gastric Banding. Obesity Surgery, 29(1), 3-14. https://doi.org/10.1007/s11695-018-3525-0

Pereira A. S. et al. (2018). Metodologia da pesquisa científica. [free e-book]. Santa Maria. Ed. UAB/NTE/UFSM

Schiavon, C. A., Bersch-Ferreira, A. C., Santucci, E. V., Oliveira, J. D., Torreglosa, C. R., Bueno, P. T., Frayha, J. C., Santos, R. N., Damiani, L. P., Noujaim, P. M., Halpern, H., Monteiro, F. L. J., Cohen, R. V., Uchoa, C. H., De Souza, M. G., Amodeo, C., Bortolotto, L., Ikeoka, D., Drager, L. F., ... Berwanger, O. (2018). Effects of bariatric surgery in obese patients with hypertension the GATEWAY randomized trial (gastric bypass to treat obese patients with steady hypertension). Circulation, 137(11), 1132-1142. https://doi.org/10.1161/CIRCULATIONAHA.117.032130

Sheng, B., Truong, K., Spitler, H., Zhang, L., Tong, X., \& Chen, L. (2017). The Long-Term Effects of Bariatric Surgery on Type 2 Diabetes Remission, Microvascular and Macrovascular Complications, and Mortality: a Systematic Review and Meta-Analysis. Obesity Surgery, 27(10), 2724-2732. https://doi.org/10.1007/s11695-017-2866-4

Sjöström, C. D., Lissner, L., Wedel, H., \& Sjöström, L. (1999). Reduction in incidence of diabetes, hypertension and lipid disturbances after intentional weight loss induced by bariatric surgery: The SOS intervention study. Obesity Research, 7(5), 477-484. https://doi.org/10.1002/j.1550-8528.1999.tb00436.x 
Research, Society and Development, v. 10, n. 12, e335101220390, 2021

(CC BY 4.0) | ISSN 2525-3409 | DOI: http://dx.doi.org/10.33448/rsd-v10i12.20390

Sjostrom, L., Narbro, K., Sjostrom, D., Karason, K., Larsson, B., Wedel, H., \& Lystig, T. (2007). Effects of Bariatric Surgery on Mortality in Swedish Obese Subjects. The New England Journal of Medicine, 357(8), 741-752

Wiggins, T., Guidozzi, N., Welbourn, R., Ahmed, A. R., \& Markar, S. R. (2020). Association of bariatric surgery with all-cause mortality and incidence of obesity-related disease at a population level: A systematic review and meta-analysis. PLoS Medicine, 17(7), 1-18. https://doi.org/10.1371/journal.pmed.1003206

Wolfe, B. M., Kvach, E., \& Eckel, R. H. (2016). Treatment of obesity. Circulation Research, 118(11), 1844-1855. https://doi.org/10.1161/CIRCRESAHA.116.307591

Zeve, J. L. de M., Novais, P. O., \& Júnior, N. D. O. (2012). Técnicas em cirurgia bariátrica: uma revisão da literatura. Ciência \& Saúde, 5(2), 132. https://doi.org/10.15448/1983-652x.2012.2.10966 